\title{
Susceptibility of Streptococcus pneumoniae to Penicillin in the State of Minas Gerais, Brazil from 1997-2004
}

\author{
Maria B. M. Bedran¹, Paulo A. M. Camargos ${ }^{1}$, \\ Geraldo Leocádio Filho², Renata M. Bedran ${ }^{1}$ \\ and Henrique C. Najar ${ }^{1}$
}

\begin{abstract}
Department of Pediatry, Medical School of Federal University of Minas Gerais ${ }^{1}$; Ezequiel Dias Foundation, State Secretary of Health of Minas Gerais²; Belo Horizonte, MG, Brazil
\end{abstract}

\begin{abstract}
We monitored the susceptibility to penicillin of invasive strains of Streptococcus pneumoniae in two reference laboratories; 502 positive cultures from patients with an active invasive infectious process were analyzed. Streptococcus pneumoniae was identified through conventional procedures, and the oxacillin disc diffusion method was used to check for penicillin susceptibility. Statistical analysis included calculations of the frequency distribution, with 95\% confidence intervals (CI), as well as chi-square tests and chi-square for linear trend for temporal analysis of susceptibility. The bacterium was isolated from patients less than a year old $(40.7 \%$ of the isolates), from infants (55.9\%), and from individuals less than 15 years old $(64.4 \%)$. The majority $(88.2 \%, 95 \% \mathrm{CI}=$ $\mathbf{8 5 . 5 \% - 9 1 . 1 \% ) ~ o f ~ t h e ~} 502$ isolates were susceptible to penicillin. There was no significant temporal trend of elevation of resistance rate during the study period $(p=0.56)$. We conclude that resistance of $S$. pneumoniae to penicillin is not yet an important clinical-epidemiological concern in the State of Minas Gerais. To provide necessary support for the adoption of therapeutic and prophylactic measures, epidemiological surveillance should be implemented at a national level to monitor the profile of susceptibility/resistance of $S$. pneumoniae to penicillin and other antimicrobials. Key Words: Streptococcus pneumoniae, antimicrobial resistance, invasive disease
\end{abstract}

Infections by Streptococcus pneumoniae significantly increase morbidity and mortality in children and the elderly, based on studies conducted internationally, and especially in developing countries, where the problem is considerably greater. This bacterium is one of the most frequent etiological pathogens in otitis, pneumonias and meningitis. [1,2] According to a WHO estimate, pneumonias are responsible for approximately 5 million child deaths per year in developing countries, $30 \%$ of which are caused by pneumococcus [1].

Received on 11 May 2005; revised 14 July 2005.

Address for correspondence: Dr. Paulo Augusto Moreira Camargos, MD, PhD. Departamento de Pediatria da Faculdade de Medicina da UFMG. Avenida Alfredo Balena 190/Sala 4061 Zip code: 30130-100 Belo Horizonte, MG Brazil. Phone: 55313248 9773.Fax:553132489664.E-mail:pcamargs@medicina.ufmg.br

The Brazilian Journal of Infectious Diseases 2005;9(5):390-397 (C) 2005 by The Brazilian Journal of Infectious Diseases and Contexto Publishing. All rights reserved.
Nowadays, there is an increasing incidence of resistance of $S$. pneumoniae to penicillin, which disrupts the treatment of infant and elderly patients with previous history of hospitalization and treatment with b-lactams [2]. This phenomenon has also been occurring with other antimicrobials, such as tetracycline, chloramphenicol, erythromycin, trimethoprimsulfamethoxazole, rifampin, and during the last decade, cephalosporins [3]. Continuous epidemiological surveillance of the susceptibility profile in infections by pneumococcus should be implemented, with periodical local and regional assessments spanning various years, allowing the establishment of strategies for control and treatment.

During the last two decades, several studies have been performed in Brazil on the susceptibility pattern of the pneumococci to penicillin and other antimicrobials. The prevalence of intermediately susceptible and resistant strains ranged from zero to $26.7 \%$ from 1981 to 2004 [4-13]. However, due to the continental dimensions of Brazil, this tremendous 
effort is still relatively limited, and it does not offer a trustworthy epidemiological view.

We examined the susceptibility pattern of $S$. pneumoniae strains to penicillin isolates between 1997 and 2004 in the state of Minas Gerais.

\section{Material and Methods}

Design, local and time period of study

We made a descriptive study, in which the susceptibility of invasive isolates of $S$. pneumoniae to penicillin was analyzed from January 1997 to December 2004. This study was conducted in the Bacteriology Section of the Medical Biology Department, Ezequiel Dias Foundation(FUNED), State Health Authority and in the Federal University of Minas Gerais (UFMG) General Hospital.

The Medical Biology Department of FUNED takes part in the Brazilian public health laboratories network, coordinated by the Ministry of Health.

\section{Inclusion and exclusion criteria}

Cultures positive for $S$. pneumoniae were collected from patients with invasive infectious illnesses in progress; one isolate was obtained per patient. Blood samples and/or cerebrospinal or pleural fluid were collected from patients, regardless of the usage of antimicrobials. Positive cultures from biological materials from other non-sterile sources were excluded from the analysis.

\section{Laboratory methods}

Streptococcus pneumoniae was identified through its typical morphology on blood agar, and chocolate agar, as indicated by Gram staining, and by susceptibility to optochin, bile solubility and alpha hemolysis [14]. The Kirby-Bauer in vitro disk diffusion method was used to determine antibiotic resistance throughout the study, using $1 \mu \mathrm{g}$ oxacillin discs, as recommended in the National Committee for Clinical Laboratory
Standards (NCCLS) [15]. Growth inhibition zones were measured after 20-24 hours of incubation. Intermediate or elevated resistance to penicillin was considered probable when the oxacillin inhibition zone size was less than $20 \mathrm{~mm}$ in diameter [16].

\section{Protocol and data collection}

The following variables were recorded in a standardized protocol, especially devised for this investigation: sex, age at diagnosis, place of residence, origin of material, institution, culture date, and antimicrobial susceptibility tests.

\section{$\underline{\text { Statistical aspects }}$}

All of the 502 isolates of $S$. pneumoniae available from the records of the two laboratories were included. Data was organized and analyzed using the software EpiInfo, version 6.04 [17]. Frequency distribution calculations were made (expressed as 95\% confidence intervals) to characterize the susceptibility/resistance of the pneumococcus to penicillin. Additionally, we used chi-square tests to compare proportions and chi-square for linear trends to determine the significance of the temporal distribution of the susceptibility/resistance. A p value $<0.05$ was considered statistically significant.

\section{Ethical aspects}

The experimental protocol was approved by the Ethics in Research Committee of the Federal University of Minas Gerais.

\section{Results}

During the period from January 1997 to December 2004, 502 invasive isolates were collected (Table 1).

The samples came from cerebral spinal fluid(69.5\%) and blood (28.8\%); most isolates were from cases of meningitis and were processed at FUNED (71.2\%). Among these samples, most (62.7\%) came from male patients. 
Age was available for $64.1 \%$ of the patients. Infants (less than 2 years old) accounted for $28.7 \%$ of the cases (95\% CI, 38.6\%-49.4\%). The age group with the highest number of isolates (23.5\%, 95\% CI, 30.9\%$41.3 \%$ ) was children younger than one year. Among the age groups, patients below the age of 15 accounted for $40.6 \%$ of the isolates positive for pneumococcus, whereas patients older than 60 years of age accounted for only $6.0 \%$. Approximately half of the isolates were patients living in Belo Horizonte, the capital of the state of Minas Gerais; there was no record of residence for $27.5 \%$ of the isolates.

The susceptibility and resistance rates of the pneumococcus isolates to penicillin during the study period were $88.2 \%$ (95\% CI, 85.5\%-91.1\%) and $11.8 \%$ (95\% CI, 9.0\%-14.6\%), respectively. The evolution of resistance rates of pneumococcus to penicillin was recorded during seven years (Table 2).

Fifty-nine isolates were resistant to penicillin. In 1997, all of the 56 strains were sensitive to this antibiotic. Between 1998 and 2004, the resistance rate ranged from 9.1 to $17.1 \%$. There was no temporal trend of increase in the resistance rate $(\mathrm{p}=0.56)$. Despite the lack of statistical significance, excluding the year 1997, during which no resistant strain was identified, the odds ratio estimates for each year in the period 1998-2004 were 1.00, 0.68, 0.89, 1.01, 0.78, 1.41 and 0.96, respectively, when compared to 1997.

Susceptibility patterns of pneumococcus to penicillin for each type of biological material were recorded (Table 3).

Approximately $70 \%$ of the susceptible strains were collected from cerebrospinal fluid. A single isolated strain in pleural liquid was classified as resistant; $11.1 \%$ and $12.2 \%$ of the isolates from cerebral spinal fluid and blood, respectively, were considered resistant.

Table 4 describes the characteristics of the 54 invasive isolates with growth inhibition zones smaller than 20mm around the oxacillin disc and therefore defined as strains of elevated and intermediate resistance to penicillin.

Among the 54 resistant isolates, 63\% were from males. Among the 43 strains resistant to penicillin from patients whose age was known, 33 of them (56\%) were collected in patients younger than two years of age (95\% CI, 51\%-79\%), indicating the importance of this type of infection for pediatric patients.

Resistant isolates from cerebrospinal fluid accounted for $69.5 \%$ of the samples. Most of the isolates (71.2\%) were processed at FUNED, due to its importance as a central laboratory in the Public Health Care System. About $56 \%$ of the resistant isolates came from the metropolitan region of Belo Horizonte.

Finally, there were no significant differences ( $\mathrm{p}>$ 0.25 ) in the distribution of susceptible versus resistant isolates by age group, sex, origin of biological material, institution, and patient place of residence.

\section{Discussion}

The susceptibility profile of $S$. pneumoniae to antibiotics can be determined through a number of methods. Determination of a minimum inhibitory concentration (MIC) constitutes the gold-standard, by which strains inhibited by concentrations inferior or equal to $0.06 \mathrm{mg} / \mathrm{mL}$ are considered susceptible to penicillin $\mathrm{G}$; strains are considered to have intermediate resistance when MIC is $0.1-1.0 \mu \mathrm{g} / \mathrm{mL}$, and they have elevated or complete resistance when these values are greater than $2 \mu \mathrm{g} / \mathrm{mL}$ [16].

Assessment of the susceptibility pattern of pneumococcus can also be determined by the disc diffusion method, which is widely available and is low in cost. It is particularly useful as a screening method and as an indicator of susceptibility to penicillin. Discs containing $1 \mu \mathrm{g}$ of oxacillin are stable and there is a high correlation between results with these discs and MIC values [17]; MIC calculation is a more complex method, which is available in very few institutions in Brazil. In the assessment of susceptibility/resistance to penicillin, the oxacillin discs gave a sensitivity greater than $99 \%$ and a specificity of $80 \%$ for MIC determination. [18]

The disc diffusion method is more sensitive (rarely are resistant strains classified as susceptible) than specific ( $6 \%$ to $40 \%$ of susceptible strains can be classified as resistant to penicillin) [17]. According to 
Table 1. Descriptive characteristics of invasive isolates of Streptococcus pneumoniae studied from 1997-2004

\begin{tabular}{lrr}
\hline Variable & $\mathbf{N}$ & $\mathbf{\%}$ \\
\hline Biological material & & \\
$\quad$ Cerebrospinal fluid & 361 & 71.9 \\
$\quad$ Blood & 139 & 30.0 \\
Others & 1 & 0.3 \\
Institution & & \\
$\quad$ FUNED & 368 & 73.3 \\
$\quad$ University Hospital & 134 & 26.7 \\
Sex & & \\
$\quad$ Male & 331 & 65.9 \\
Female & 171 & 34.1 \\
Age group (years) & & \\
$\quad$ < 1 & 118 & 23.5 \\
1-2 & 26 & 5.2 \\
3-15 & 60 & 11.9 \\
16-60 & 94 & 18.7 \\
$\quad$ 60 & 30 & 6.0 \\
$\quad$ Unknown & 174 & 34.7 \\
Patient location of residence & & \\
$\quad$ Metropolitan region of Belo Horizonte & 248 & 49.4 \\
Countryside & 116 & 23.1 \\
$\quad$ Unknown & 138 & 27.5 \\
Susceptibility to penicillin G & & \\
Susceptible & & \\
Resistant & 443 & 88.2 \\
& 59 & 11.8 \\
\hline
\end{tabular}

Table 2. Pattern of susceptibility/resistance of Streptococcus pneumoniae to penicillin G through the disc diffusion method in 502 invasive isolates

\begin{tabular}{lcccc}
\hline Year & Susceptible N (\%) & Resistant* N (\%) & Total N (100\%) \\
\hline 1997 & $56(100.0)$ & $0(0.0)$ & 56 \\
1998 & $71(87.7)$ & $10(12.3)$ & 81 \\
1999 & $58(82.9)$ & $12(17.1)$ & 70 \\
2000 & $63(86.3)$ & $10(13.7)$ & 73 \\
2001 & $72(87.8)$ & $10(12.2)$ & 82 \\
2002 & $39(84.8)$ & $7(15.2)$ & 46 \\
2003 & $50(90.9)$ & $5(9.1)$ & 55 \\
2004 & $34(87.2)$ & $5(12.8)$ & 39 \\
Total & $443(88.2)$ & $59(11.8)$ & 502 \\
\hline
\end{tabular}

*indicates intermediate and elevated resistance 
Table 3. Origin and susceptibility of Streptococcus pneumoniae to penicillin through the disc diffusion method from 1997-2004

\begin{tabular}{lrrrr}
\hline Source & \multicolumn{2}{c}{ Susceptible } & \multicolumn{2}{c}{ Resistant } \\
\hline & N & \% & N & \% \\
\hline Cerebrospinal fluid & 310 & 71.5 & 41 & 69.5 \\
Blood & 123 & 28.5 & 17 & 28.8 \\
Pleural fluid & 0 & 0 & 1 & 1.7 \\
Total & 433 & 100.0 & 59 & 100.0 \\
\hline
\end{tabular}

Table 4. Descriptive characteristics of the 54 invasive isolates of Streptococcus pneumoniae resistant to penicillin studied from 1997-2004

\begin{tabular}{lcc}
\hline Variable & $\mathbf{N}$ & $\mathbf{\%}$ \\
\hline Sex & & \\
$\quad$ Male & 37 & 62.7 \\
$\quad$ Female & 22 & 37.3 \\
Age group (years) & & \\
$\quad<1$ & 24 & 40.7 \\
$1-2$ & 9 & 15.2 \\
$3-15$ & 5 & 8.5 \\
$16-60$ & 12 & 20.3 \\
$\quad$ 60 & zero & Zero \\
$\quad$ Unknown & 9 & 15.3 \\
Material & & \\
$\quad$ Cerebrospinal fluid & 41 & 69.5 \\
$\quad$ Blood & 17 & 28.8 \\
$\quad$ Pleural fluid & 1 & \\
Institution & & 71.2 \\
$\quad$ FUNED & 42 & 28.8 \\
$\quad$ University Hospital & 17 & 55.9 \\
Local of residence & & 28.9 \\
$\quad$ City of Belo Horizonte & 33 & 15.2 \\
$\quad$ Countryside & 17 & \\
$\quad$ Unknown & 9 & \\
\hline
\end{tabular}


the American Academy of Pediatrics [19], disc diffusion reliably identifies every susceptible pneumococcus (growth inhibition zones equal or greater than $20 \mathrm{~mm}$ ). However, it does not allow distinction between strains with complete resistance from those with intermediate resistance to penicillin.

In Brazil, a continental-scale country, there are relatively few studies assessing susceptibility patterns of pneumococcus. During the last 23 years, in an examination of studies with invasive samples, resistance rates have ranged from 0 to $25 \%$ [4-13]. The state of Minas Gerais has an area almost the size of France, and though it is only one state, our sample is larger than in the previous studies. In a comparison with several other Brazilian states, the state of Minas Gerais is nowadays in an intermediate epidemiological situation (11.7\% resistance), especially after 1998, the year in which the resistance was $12.3 \%$ [11].

Kertesz et al. conducted a prospective multicentric study (named SIREVA Project) in six Latin American countries, including Brazil; 1,649 invasive samples from children younger than five years of age were studied. The proportion of isolates resistant to penicillin ranged from $12 \%$ in Colombia to $47.3 \%$ in Mexico, where the highest levels of complete penicillin resistance were reported (21.3\%). The mean rate of resistance in the six countries was $24.9 \%$, including $8.2 \%$ elevated resistance [20]. In a comparison with North American countries, the rates found in our study were lower than those found in Canada (30.2\%) [21], the United States (43.8\%) [21] and Mexico (48\%) [20].

The rates of resistance observed in some European countries, such as Spain (58\%) [22], France (32\%) [23], and Hungary (58\%) [24] were much higher than those we found in the state of Minas Gerais. The tendency was reversed when our results were compared with levels from Germany (0.8\%) [25], Sweden (2.0\%) [26], and Italy (5.5\%) [27].

Among Asian countries, Korea has the world's highest rate of resistance, involving more than 70\% of the isolates, among which $33 \%$ presented complete resistance to penicillin [28]. In a retrospective study conducted in that country from 1985-1996, resistance rates in isolates from children younger than 12 years of age with invasive infections ranged from zero in 1989 to $89 \%$ in 1995 , while the rate of resistance in 1996 was 69\% [28].

The resistance rate to penicillin in our study was highest among infants (59.2\%), similar to what is known from other studies $[6,9,20]$, probably due to a greater utilization of broad-spectrum antimicrobials and a higher prevalence of pneumococcic infections in patients of that age group. Similar results were reported by Ko et al. [11], who have found that $71.0 \%$ of isolates from infants were resistant to penicillin. These findings suggest that more attention should be given to this age group.

A map of the profile of resistance/susceptibility of $S$. pneumoniae should be made through studies similar to ours, which ideally should include determination of MIC as well as implementation of an effective epidemiologic surveillance system with continuous monitoring of resistance levels. This would require a network of laboratories in charge of performing the available microbiological tests for all invasive isolates. The diffusion method in oxacillin discs should be used as a screening method, and in the following phase the resistant isolates should submitted to conventional MIC determination, or the E-test should be applied, which is a more practical and rapid method, with virtually $100 \%$ agreement with MIC [29].

The systematic use of blood cultures in the routine care of children with pneumonia and meningitis, especially among those younger than five years of age, is of paramount importance and would contribute effectively towards the recognition of local/regional/national patterns of resistance, allowing for the development of strategies of pneumococcal infection control.

In order to reduce the risk of colonization and dissemination, and the development of disease due to resistant strains in communities, preventive measures should include anti-pneumococcic vaccination of all children and the judicious usage of antimicrobials, especially $\beta$-lactams [30,31]. 


\section{References}

1. World Health Organization. Technical basis for WHO recommendations on the management of pneumonia in children at first level health facilities. Document WHO/ ARI/91-120,1991.

2. Caputo G.M., Appelbaum P.C., Liu H.H. Infections due to penicillin resistant pneumococci. Clinical epidemiological and microbiological features. Arch Intern Med 1993;153:1301-10.

3. Bradley J.S., Connor J.D. Ceftriaxone failure in meningitis caused by Streptococcus pneumoniae with reduced susceptibility to beta lactam antibiotics. Pediatr Infect Dis J 1991;10:871-3.

4. Teixeira L.M. Serotypes and antimicrobial susceptibility of Streptococcus pneumoniae isolated in Rio de Janeiro, Brasil. Rev Microbiol 1988;19:93-9.

5. Sossegolo J.F., Levin A.S.S., Levy C.E., et al. Distribution of serotypes and antimicrobial resistance Streptococcus pneumoniae strains isolated in Brazil from 1988-1992. J Clin Microbiol 1994;32:906-11.

6. Berezin E.N., Carvalho E.S., Casagrande S., et al. Streptococcus pneumoniae penicillin nonsusceptible strains in invasive infections in São Paulo, Brazil. Pediatr Infect dis J 1996;15:1051-3.

7. Levin A.S.S., Teixeira L.M., Sossegolo J.F., Barone A.A. Resistance of Streptococcus pneumoniae to antimicrobials in São Paulo, Brazil: clinical features and serotypes. Rev Inst Med Trop S Paulo 1996;38:187-92.

8. Teixeira L.M., Carvalho M.G.S., Castineiras T.M.P.P., et al. Serotyping distribution and antimicrobial resistance of Streptococcus pneumoniae isolated in Brazil (19921996). Adv Exp Med Biol 1997;418:269-71.

9. Brandileone M.C.C., Vieira V.S.D., Casagrande T.S., et al. Prevalence of serotypes and antimicrobial resistance of Streptococccus pneumoniae strains isolated from Brazilian children with invasive infections. Microbial Drug Resistance 1997;3:141-6.

10. Brandileone M.C.C., Vieira U.S.D., Casagrande S.T., et al. Characteristics of isolates Streptococcus pneumoniae from middle aged and elderly adults in Brazil: capsular serotypes and antimicrobial sensitivity with invasive infections. Braz J Infect Dis 1998;2:90-6.

11. Ko A.I., Reis J.N., Copolla S.J., et al. Clonally related penicillin-nonsusceptible Streptococcus pneumoniae serotype 14 from cases of meningitis in Salvador, Brazil.Clin Infect Dis 2000;30:78-86.

12. Nascimento-Carvalho C.M., Souza L.S.F., Carvalho O.A.M., et al. Cepas invasivas de pneumococo isoladas de crianças e adolescentes em Salvador. J Pediatr (Rio J) 2004;79:209-14.
13. Mantese O.C., Paula A., Moraes A.B., et al. Prevalência de sorotipos e resistência antimicrobiana de cepas do Streptococcus pneumoniae. J. Pediatr (Rio J) 2004;79:537-42.

14. Ruoff K.L., Whisley R.A., Beighton D. Streptococcus. In: Murray PR, Baron EJ, Pfaller MA, Tonover FC, Yolken RH, Manual of Clinical Microbiology. $7^{\text {th }}$ ed. ASM Press; 1999. p.283-96.

15. National Committee for Clinical Laboratory Standards 1995. Performance standards for antimicrobial susceptibility tests: approved standard. NCCLS Publication M2-A5. Villanova, PA: National Committee for Clinical Laboratory Standards; 1997.

16. Mantese O.C. Pneumococo resistente à penicilinaimplicações práticas. J Pediatr 1999; 75:74-90.

17. Dean A.G., Dean J.A., Coulombier D., et al. Epi Info, Version 6.04: a Word Processing. Database, and Statistics Program for Epidemiology on Microcomputers. 1994. Center for Disease Control and Prevention, Atlanta.

18. Bartlett J.C., Breiman R.F., Mandell L.A., File T.M. Jr. Community acquired pneumonia in adults: Guidelines for Management. Clin Infect Dis 1998;26:811-38.

19. American Academy of Pediatrics. Committee on Infectious Diseases. Therapy for children with invasive pneumococcal infections. Pediatr 1997;99:289-99.

20. Kertesz D.A., Di Fábio J.L., Brandileone M.C.C. Invasive Streptococcus pneumoniae infeccion in Latin American children: results of Pan American Health Organization surveillance study. Clin Infect Dis 1998;26:1355-61.

21. Doern G.V., Pfaller M.A., Kugler R., et al. Prevalence of antimicrobial resistance among respiratory tract isolates of Streptococcus pneumoniae in North America 1997. Results from the Sentry Antimicrobial Surveillance Program. Clin Infect Dis 1998;27:764-70.

22. Ramos J.T., Saavedra J., Ruiz-Contreras J., et al. Invasive antibiotic-resistant Streptococcus pneumoniae in children in Madrid. Pediatr Infect Dis J 1998;17:252-55.

23. Geslin P. Bêta-lactamines et pneumocoques multirésistants isolés en France (1984-1994). Med et Hyg 1995;53:2111-18.

24. Marton A., Gulyas M., Munoz R., Tomasz A. Extremely high incidence of antibiotic resistance in clinical isolates of Streptococcus pneumoniae in Hungary. J Infect Dis 1991,163:542-8.

25. Reinert R.R., Kaufhold A., Schlaeger J.J., et al. Serotype distribution and antibiotic susceptibility of Streptococcus pneumoniae isolates causing systemic infectious among children in Germany, 1992 to 1996. Pediatr Infect Dis J 1997; 16:244-5.

26. Hedlund J., Svenson S.B., Kalin M., et al. Incidence, capsular types and antibiotic susceptibility of invasive Streptococcus pneumoniae in Sweden. Clin Infect Dis 1995;21:948-53. 
27. Marchese A., Debbia A.E., Arvigo A., et al. Susceptibility of Streptococcus pneumoniae strains isolated in Italy to penicillin and ten other antibiotics. J Antimicr Chemother 1995;36:833-7.

28. Choi E.H., Lee H.J. Clinical outcome of invasive infectious by penicillin resistant Streptococcus pneumoniae in Korean children. Clin Infect Dis 1998;26:1346-54.

29. Jorgensen J.H., Ferraro M.J. Antimicrobial susceptibility testing: general principles and contemporary practices. Clin Infect Dis 1998;26:978-80.

30. Dowell S.F., Marcy M., Phillips W.R., et al. Principles of judicious use of antimicrobial agents for pediatric upper respiratory tract infections. Pediatrics 1998;101:163-5.

31. Pichichero M.E. Short course antibiotic therapy for respiratory infections: a review of the evidence. Pediatr Infect Dis J 2000;19:929-37. 